\title{
Transvenous pacemaker electrodes placed unintentionally in the left ventricle: three cases
}

\author{
S.J. Winner and N.A. Boon* \\ Department of Cardiovascular Medicine, John Radcliffe Hospital, Oxford OX3 9DU, UK.
}

\begin{abstract}
Summary: Three patients are described in whom pacemaker electrodes were unintentionally placed within the left ventricle, followed by considerable delay before the error was recognized. In two cases temporary pacemaker wires were inserted into the subclavian artery and passed along a retrograde course. One patient required urgent surgery for acute arterial obstruction on removal of the wire. In the third case, a permanent wire was inserted correctly into a vein but traversed the atrial septum, probably via a patent foramen ovale, to enter the left ventricle. Twelve lead electrocardiograms in all three patients showed paced complexes with right bundle branch block configuration. This appearance should raise suspicion that the pacemaker electrode might be in the left ventricle, in which case its position should be defined by chest radiographs (including a lateral view) and echocardiography.
\end{abstract}

\section{Introduction}

We report three patients in whom pacemaker electrodes were unintentionally placed within the left ventricle, followed by considerable delay before the error was recognized.

\section{Case reports}

\section{Patient 1}

A 70 year old woman was admitted to hospital following several episodes of syncope. A 24-hour electrocardiogram (ECG) showed sinus rhythm, with passages of 2:1 atrioventricular block, and periods of sinus arrest up to three seconds in duration. A temporary pacing wire was inserted via a right supraclavicular approach. She remained well, and was transferred to the Regional Cardiac Centre 5 days later. A twelve lead ECG showed paced beats with right bundle branch block configuration. A posteroanterior (PA) chest radiograph showed that the pacemaker wire followed an odd course, with the tip in an abnormally lateral position within the cardiac shadow. A permanent pacemaker was implanted on the next day, and immediately afterwards the temporary wire was withdrawn.

Correspondence and present address: S.J. Winner, M.A., M.B., B.Chir., M.R.C.P., University Division of Geriatric Medicine, The Radcliffe Infirmary, Oxford OX2 6HE.

* Present address: Department of Cardiology, Royal Infirmary of Edinburgh, Edinburgh EH3 9YW, UK.

Accepted: 17 August 1988
Post-operatively, the right brachial pulse weakenfed, and the forearm became cool. It was then recognized that the tip of the temporary pacemaker had ben placed in the left ventricle, via the right subclavian artery and aorta, and that removal of the wir caused traumatic occlusion of the artery. An arteriogram (Figure 1) demonstrated occlusion of the subclavian artery, which tapered from a point distal to the origin of the vertebral artery; collatesal vessels from around the shoulder joint filled the axillary artery. The brachial artery was also occlud\&d, with appearances suggesting an embolus at the m $\overrightarrow{\mathrm{f}} \mathrm{d}$ point of the humerus.

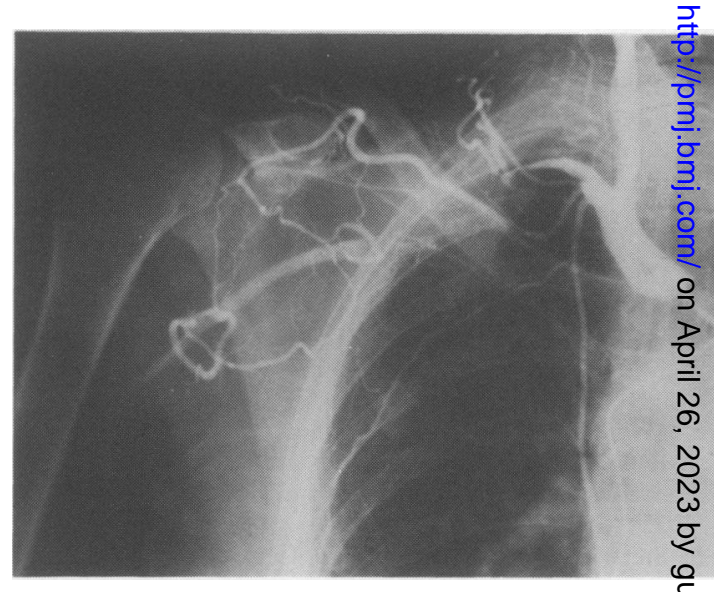

Figure 1 Patient 1: arteriogram showing occlusion of ghe right subclavian artery following withdrawal of the temporary pacemaker wire.

(C) The Fellowship of Postgraduate Medicine, 1 
Despite therapy with intravenous heparin, paraesthesiae and reduced sensation developed in the right forearm, and the brachial and radial pulses disappeared. Urgent surgery was undertaken. The right subclavian artery was found to be thrombosed, with intimal damage. An end-to-end goretex graft was inserted between the subclavian and axillary arteries, and distal thrombus was extracted using a Fogarty embolectomy catheter. Initial progress was satisfactory, and she was symptom-free with good peripheral pulses. However, 16 months later she reported ischaemic symptoms on exercising the right arm, and the peripheral pulses were no longer palpable. Arch aortography demonstrated occlusion of the graft. Further surgery is under consideration.

\section{Patient 2}

An 80 year old man was admitted to hospital following the acute onset of breathlessness, confusion, and

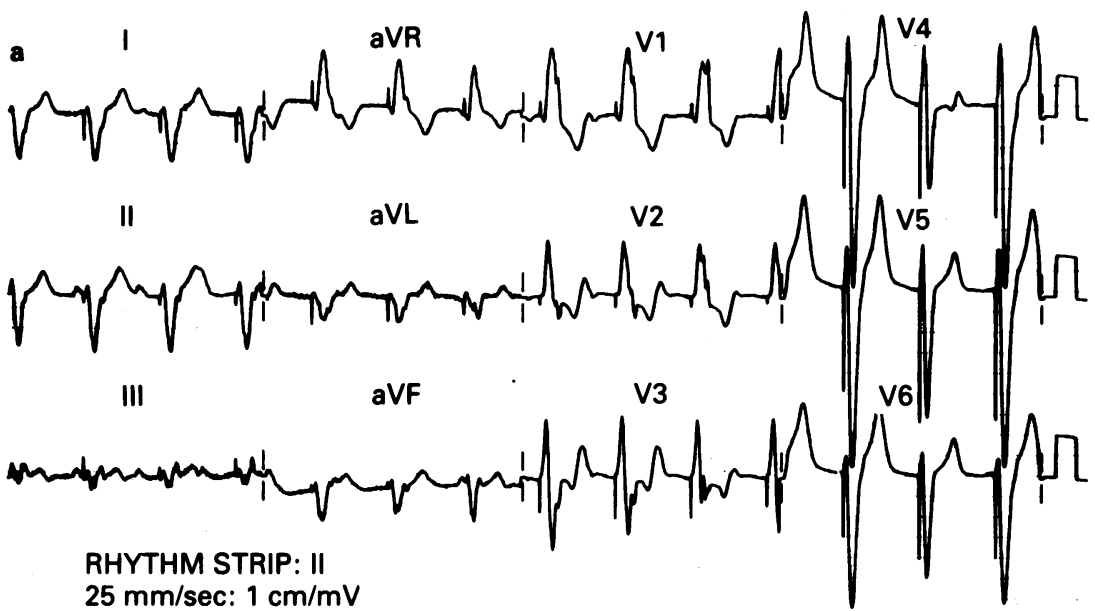

RHYTHM STRIP: ॥

$25 \mathrm{~mm} / \mathrm{sec}: 1 \mathrm{~cm} / \mathrm{mV}$
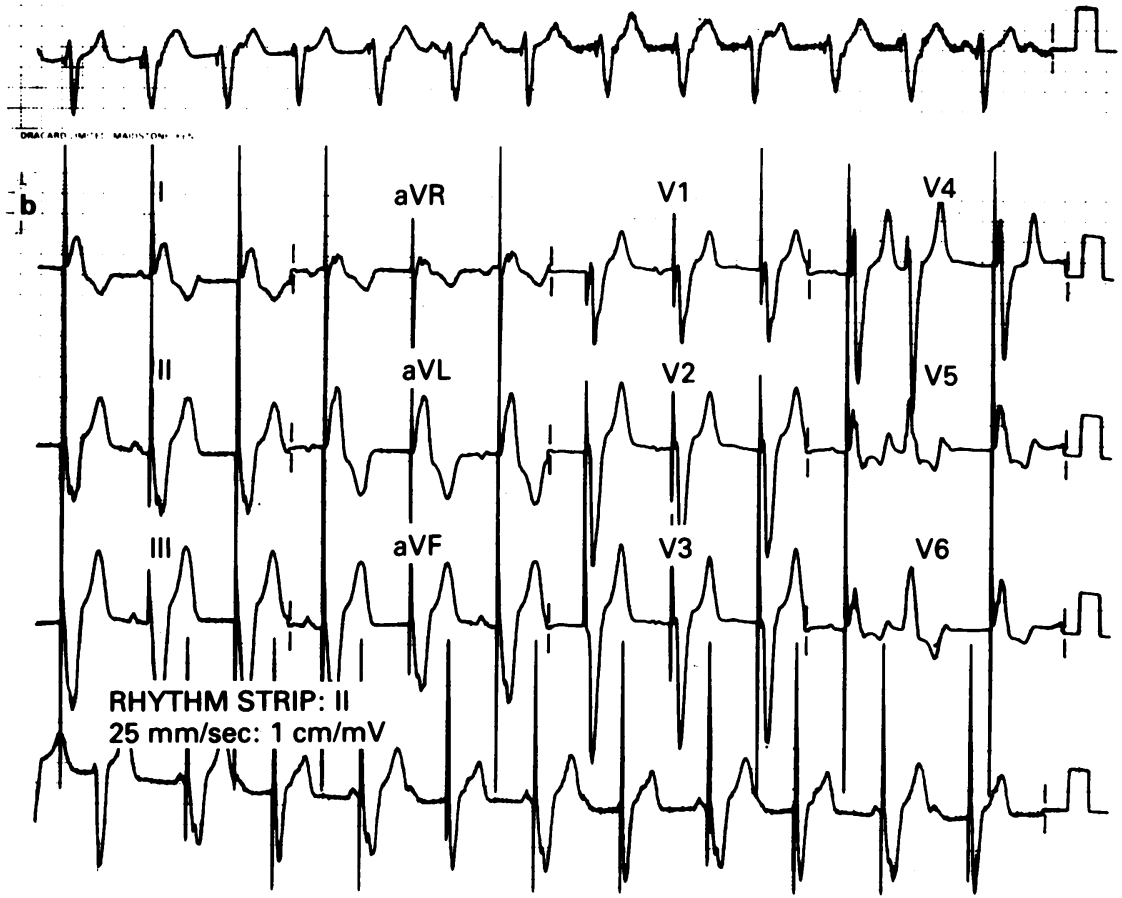

Figure 2 Patient 2: twelve lead electrocardiograms. (a) Temporary pacemaker in the left ventricle, paced beats with right bundle branch block pattern. (b) Two days later, permanent pacemaker in the right ventricle, paced beats with left bundle branch block pattern. 
impaired vision on looking to the left. Examination revealed bradycardia and left homonymous hemianopia; an ECG showed complete heart block. A temporary pacemaker was inserted via a left infraclavicular approaich, after attempts to insert a wire from the right side had failed.

Six days later he was transferred to the Regional Cardiac Centre. An ECG showed paced beats with right bundle branch block configuration (Figure 2). Antero-posterior and lateral chest radiograph appearances (Figure 3) suggested that the tip of the pacemaker was in the left ventricle. On the next day, a permanent pacemaker was implanted. X-ray screening during this procedure confirmed that the temporary wire lay within the left ventricle. After consultation with a vascular surgeon, the temporary wire was withdrawn. Arterial bleeding occurred at the skin puncture site, and was controlled by pressure. There was temporary loss of the left brachial and radial pulses; the former returned in 5 minutes, the latter after 60 minutes. A cranial computed tomographic (CT) scan showed a slightly haemorrhagic infarct in the right temporo-occipital region, with a mature infarct in the left posterior frontal region, and others in the basal ganglia on both sides. His confusion rapidly resolved, and the hemianopia showed some improvement.

\section{Patient 3}

A 72 year old woman was admitted to hospital with a 5-week history of dizzy spells, and five episodes of loss of consciousness. Five years previously, she had been noted to have transient complete heart block, during an episode of myocarditis attributed to viral infection. An ECG showed sinus rhythm with first degree heart block, and frequent short-lived episodes of complete heart block. A temporary pacemaker was inserted and she was transferred to the Regional Cardiac Centre.

A permanent pacemaker wire was inserted via a cut-down to the left cephalic vein, with a pacing threshold of 0.7 volts. The temporary wire was removed. Two days later, she suffered sudden and transient loss of consciousness when attempting to stand up after using a commode. There were no abnormal physical signs, her pacemaker was found to be functioning satisfactorily, and the episode was attributed to vaso-vagal syncope. She remained well until one year later, when she was admitted to hospital with left subclavian vein thrombosis, confirmed by venography. This was treated successfully with intravenous streptokinase, followed by heparin, and oral anticoagulation with warfarin.

Two years after permanent pacemaker insertion, her radiographs were examined in the course of a research project. The permanent pacemaker wire followed an abnormal route after entering the right
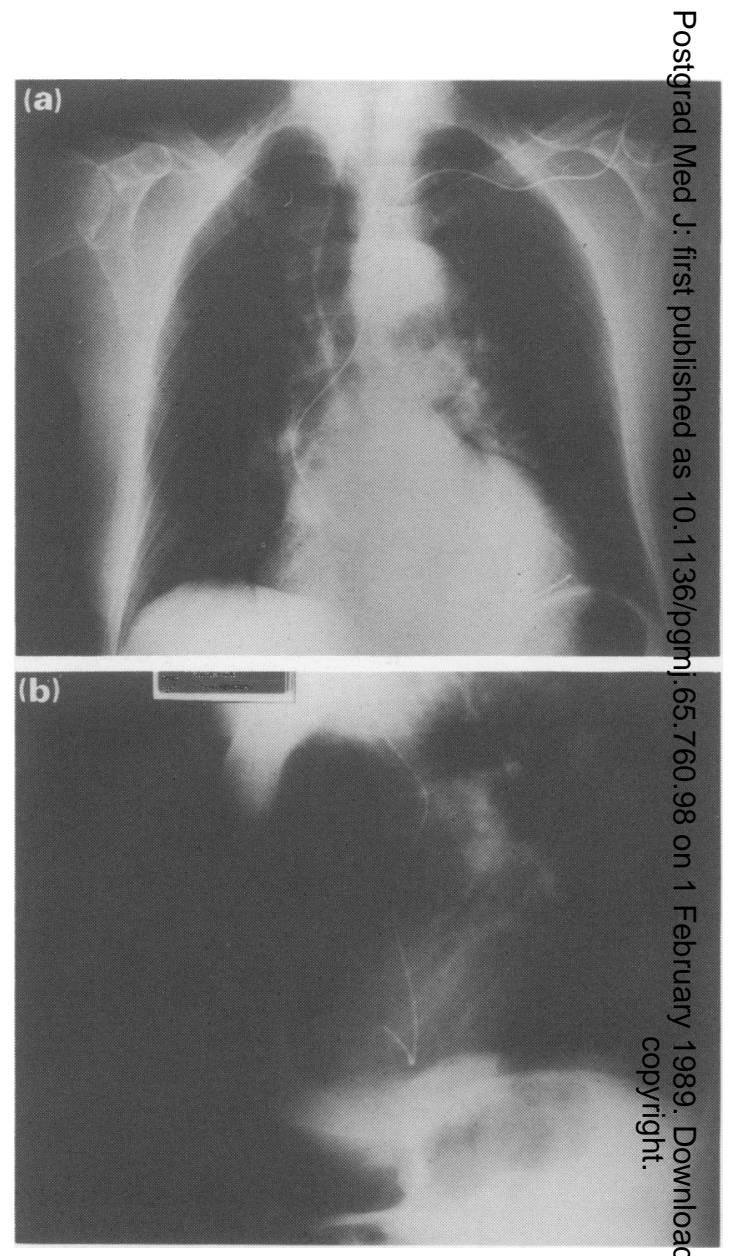

Figure 3 Patient 2: (a) antero-posterior and (b) la $\stackrel{0}{\text { eral }}$ chest radiographs showing temporary pacemaker electrode placed in the left ventricle via the left subclapian artery.

atrium, consistent with passing across the atrial 氶ptum, through the mitral valve and into the tifeft ventricle. The patient was reviewed and found tobe well. An ECG showed paced complexes with comptete right bundle branch block configuration. T:Wodimensional echocardiography (Figure 4) confirroed that the wire ran across the atrial septum and mitral valve, and lay with its tip in the left ventriele. Apart from this abnormal position, pacemaker fupction was normal. Withdrawal of the wire was got attempted, for fear of precipitating syste ic embolism. She has remained well, on long-term warfarin therapy.

\section{Discussion}

In each of these patients, a pacemaker electrodi intended for the right ventricle was placed in the tef 

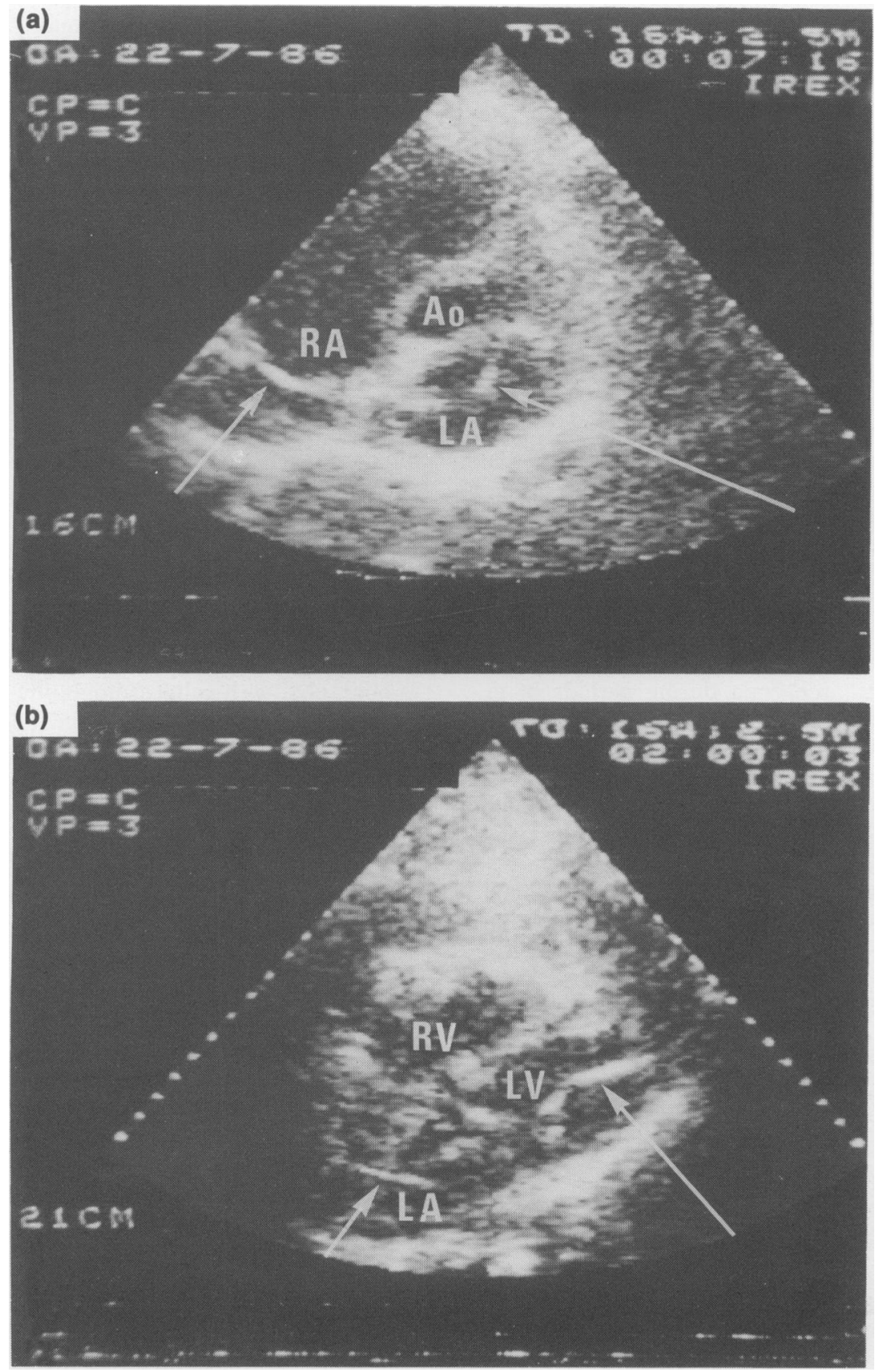

Figure 4 Patient 3: two-dimensional echocardiogram. (a) Parasternal short axis view. (b) Subcostal four chamber view. Ao, aorta. RA, right atrium. LA, left atrium. LV, left ventricle. Arrows point to pacemaker wire, seen in (a) passing across the inter-atrial septum, probably via a patent foramen ovale, and in (b) passing from LA to LV. 
ventricle, and the error was not appreciated at the time of the procedure. In two patients, the subclavian artery was mistaken for the vein, and a temporary pacemaker wire was passed along a retrograde course through the artery, the aorta, and the aortic valve. We are not aware of any published reports of similar cases. Puncture of the subclavian artery is the most frequent complication of attempted subclavian vein catheterization, occurring in approximately $3 \%$ of attempts, but in most cases the operator is immediately aware of the error. ${ }^{1}$

Our first case (Patient 1) was diagnosed only when 'routine' withdrawal of the intra-arterial temporary wire led to catastrophic acute arterial obstruction. The increased awareness resulting from this episode probably contributed to the prompt diagnosis of the second case (Patient 2), who was admitted 2 years later. On this occasion, withdrawal of the wire did not give rise to complications apart from temporary loss of peripheral pulses, attributed to arterial spasm rather than embolism. This might not have been noticed in the absence of specific monitoring. Some of the cerebral infarcts shown on this patient's cranial CT scan may have been caused by thrombotic emboli arising from the wire. However, homonymous hemianopia was noted before insertion of the temporary pacemaker, and other causes were implicated, including cerebral ischaemia during periods of extreme bradycardia or asystole complicating his complete heart block.

In the third patient, a permanent wire was inserted correctly into a vein and advanced to the right atrium, but then traversed the atrial septum, probably via a patent foramen ovale, to enter the left ventricle. The foramen ovale remains a potential opening, valvular or 'probe patent', in a quarter of the adult population. ${ }^{2}$

\section{References}

1. Sznajder, J.I., Zveibil, F.R., Bitterman, H., Weiner, P. \& Bursztein, S. Central vein catheterization. Failure and complication rates by three percutaneous approaches. Arch Intern Med 1986, 146: 259-261.

2. Hagen, P.T., Scholz, D.G. \& Edwards, W.D. Incidence and size of patent foramen ovale during the first 10 decades of life: an autopsy study of 965 normal hearts. Mayo Clin Proc 1984, 59: 17-20.

3. Judson, P.L., Moore, T.B., Swank, M. \& Ashworth, H.E. Two-dimensional echocardiograms of a transvenous left ventricular pacing catheter. Chest 1981, 80: 228-230.

4. Schiavone, W.A., Castle, L.W., Salcedo, E. \& Graor, R. Amaurosis fugax in a patient with a left ventricular endocardial pacemaker. Pace 1984, 7: 288-292.
Two similar cases have been reported previous Friable debris was seen on the wire at surgical removîn in one, and amaurosis fugax was the presentin symptom in the other. ${ }^{3,4}$ This potential complication of permanent transvenous pacemaker insertion is not well known, and was not mentioned in a rece review. ${ }^{5}$

Our patient was diagnosed when a research projeg prompted detailed scrutiny of the chest radiograph Warfarin therapy prescribed for her venous throm bosis may have prevented systemic emboli from arising. None of the clinical events in this case can be attributed unequivocally to the misplaced wire, an this 'benign' course suggests the possibility that there may be other as yet unrecognized patients witg permanent pacemaker electrodes in the left ventricle

Pacing with an intracardiac electrode near the apex of the right ventricle commonly produces QRS cong. plexes on an electrocardiogram which resemble left bundle branch block. ${ }^{6,7}$ In all three of our patients, 8 twelve lead ECG showed paced beats with the configuration of complete right bundle branch blocf. Although this is an occasional finding with a right ventricular electrode, ${ }^{7,8}$ such appearances should sug $\overrightarrow{g_{7}}$ gest the possibility that the pacemaker tip has inadvertently been inserted into the left ventricle. It: position should be defined by PA and lateral che radiographs, with echocardiography where appropriate.

\section{Acknowledgements}

We are grateful to Professor P. Sleight for allowing us report on patients under his care, and to Dr C. Woodham for radiological advice.
5. Phibbs, B. \& Marriott, H.J.L. Complications of perma nent transvenous pacing. $N$ Engl $J$ Med 1985, 31: $1428-1432$.

6. Goldman, M.J. Principles of Clinical Electrocardiograph Lange Medical Publications, California, 1979, p. 303.

7. Barold, S.S., Narula, O.S., Javier, R.P., Linhart, J.W? Lister, J.W. \& Samet, P. Significance of right bundle्हु branch block patterns during pervenous ventricular pae ing. Br Heart J 1969, 31: 285-290.

8. Mower, M.M., Aranaga, C.E. \& Tabatznik, B. Unusual patterns of conduction produced by pacemaker stimu伿 Am Heart $J$ 1967, 74: 24-28. 